\title{
ASSOCIAÇÃO DE EXTRATOS VEGETAIS E TERRA DE DIATOMÁCEA NO CONTROLE DO GORGULHO-DO-MILHO Sitophilus zeamais MOTS. (COLEOPTERA: CURCULIONIDAE)
}

\author{
Leandro do Prado Ribeiro ${ }^{1 *}$, José Djair Vendramim²
}

\begin{abstract}
RESUMO - A integração de estratégias é uma prática essencial para se obter sucesso no controle de insetospraga, configurando-se na base de qualquer programa de manejo integrado de pragas (MIP). Diante desse contexto, a combinação de pós inertes com inseticidas naturais pode se configurar em uma estratégia interessante para potencializar a ação inseticida, por meio de possíveis efeitos aditivos ou sinérgicos. Considerando esses aspectos, o presente estudo teve como objetivo avaliar a associação de extratos orgânicos brutos de sementes de duas espécies de anonáceas (Annona mucosa Jacq. e Annona montana Macfad.) com terra de diatomácea no controle de Sitophilus zeamais Mots. (Coleoptera: Curculionidade), em milho armazenado. A utilização conjunta de terra de diatomácea e extratos de sementes de A. mucosa, em hexano, e de A. montana, em diclorometano, independentemente da concentração utilizada ( $\mathrm{CL}_{30}$ ou $\mathrm{CL}_{50}$ previamente ajustada), ocasionam efeitos inseticidas ou protetores de grãos equivalentes ou mesmo inferiores em comparação ao emprego desses tratamentos isoladamente, o que demonstra efeito antagônico entre as duas técnicas.
\end{abstract}

Palavras chave: acetogeninas, aleloquímicos, Annonaceae, grãos armazenados, MIP.

\section{ASSOCIATION OF VEGETABLE EXTRACTS AND DIATOMACEOUS EARTH IN THE CONTROL OF Sitophilus zeamais MOTS. (COLEOPTERA: CURCULIONIDAE)}

\begin{abstract}
The integration of strategies is an essential practice for successful pest insect control, forming the basis of any integrated pest management (IPM) program. In this context, the combination of inert dusts with natural insecticides can be an interesting strategy to potentiate the insecticidal action by means of possible additive and synergistic effects. Considering these aspects, the objective of this study was to evaluate the association of crude organic extracts prepared from seeds of two Annonaceae species (Annona mucosa Jacq. and Annona montana Macfad.) with diatomaceous earth in the control of Sitophilus zeamais Mots. (Coleoptera: Curculionidade) in stored corn. The associated use of diatomaceous earth and A. mucosa hexanic seed extract and A. montana dichloromethane seed extract, regardless of the concentration used, regardless of the concentration used ( $L C_{30}$ or $L C_{50}$ previous estimated), cause equivalent insecticidal or grain-protective effects or even lower when these are employed in isolation, which shows an antagonistic effect between the two techniques.
\end{abstract}

Keywords: acetogenins, Allelochemicals, Annonaceae, IPM, stored pest.

\footnotetext{
${ }^{1}$ Pesquisador, Centro de Pesquisa para Agricultura Familiar, Empresa de Pesquisa Agropecuária e Extensão Rural de Santa Catarina (CEPAF/EPAGRI), Chapecó, Santa Catarina, Brasil, leandroribeiro@epagri.sc.gov.br.

${ }^{2}$ Professor, Escola Superior de Agricultura "Luiz de Queiroz", Universidade de São Paulo (ESALQ/USP), Piracicaba, São Paulo, Brasil, jdvendra@usp.br.
} 


\section{INTRODUÇÃO}

A integração de estratégias é uma prática essencial para se obter sucesso no controle de insetos-praga, configurando-se na base de qualquer programa de manejo integrado de pragas aplicável aos grãos armazenados (Hagstrum \& Phillips, 2017). Em nível mundial, tal estratégia tem-se tornado ainda mais importante em virtude do crescente número de populações de insetos resistentes aos inseticidas sintéticos (Boyer et al., 2012), a preocupação dos consumidores com os níveis de resíduos de xenobióticos nos produtos a serem consumidos (Rumbos et al., 2018) e a necessidade de alternativas eficientes de manejo aplicáveis às condições tropicais (Ribeiro et al., 2016). Nesse contexto, o uso de protetores de grãos, especialmente de pós inertes à base de terra de diatomácea, e de derivados botânicos com ação inseticida/insetistática, são alternativas de manejo amplamente pesquisadas e difundidas para o manejo de pragas de grãos armazenados (Malia et al., 2016; Hikal et al., 2017).

A utilização de pós inertes, técnica utilizada pelos agricultores de subsistência antes do advento dos inseticidas sintéticos, tem ressurgido como uma alternativa importante e promissora no controle de insetos-praga em grãos armazenados (Shah \& Khan, 2014). A terra de diatomácea (sedimento de carapaças de algas diatomáceas) é o pó inerte mais estudado, sendo utilizado para o controle de insetos de grãos armazenados em vários países como Austrália, Canadá e Estados Unidos (Atui et al., 2003). No Brasil, há formulações disponíveis comercialmente com eficácia reportada sobre diferentes espécies de insetos-praga incidentes em condições tropicais (Cerutti \& Lazzari, 2005; Marsaro Júnior et al., 2007; Ribeiro et al., 2008; Ribeiro et al., 2018). Não obstante, com a diminuição da disponibilidade de moléculas sintéticas com ação inseticida pela retirada do mercado de compostos de amplo espectro de atividade, tem-se estimulado, novamente, o estudo de inseticidas botânicos como alternativa no controle de insetos-praga de grãos armazenados, dada sua maior especificidade de ação sobre o organismo-alvo, menor toxicidade e risco à saúde dos aplicadores assim como rápida degradabilidade, o que diminui a presença de resíduos no ambiente e nos produtos tratados (Isman, 2006). Além disso, os inseticidas botânicos são constituídos por vários compostos biologicamente ativos, o que diminui a possibilidade de seleção de populações resistentes pelos variados modos de ação em um mesmo produto (Rosell,
2008). Em virtude da facilidade de obtenção de derivados botânicos (nas formas de pós vegetais, extratos ou óleos), tal ferramenta tem sido utilizada no controle de espéciespraga de grãos armazenados em vários países da América Latina, África e Ásia (Oliveira \& Vendramim, 1999).

Entre as famílias botânicas de ocorrência na região neotropical, Annonaceae é uma das fontes de moléculas bioativas mais promissoras em virtude de sua grande diversidade de metabólitos secundários com atividade biológica, como alcaloides, acetogeninas, terpenoides e lactonas (Pinheiro et al., 2009). Os efeitos promissores de extratos orgânicos brutos obtidos das sementes de Annona montana Macfad. e Annona mucosa Jacq. no manejo do gorgulho-do-milho, Sitophilus zeamais Mots. (Coleoptera: Curculionidae), principal espécie-praga do milho armazenado (Cerutti \& Lazzari, 2005), foram demonstrados por Ribeiro et al. (2013) e Ribeiro et al. (2014). A atividade biológica de tais derivados decorre da ação sinergística de acetogeninas estruturalmente diversificadas (Ansante et al., 2015; Souza et al., 2017).

Dentro dos preceitos de um programa de manejo integrado de pragas aplicável aos grãos armazenados (MIP Grãos), a combinação de pós inertes com inseticidas naturais pode se configurar em uma estratégia interessante para potencializar o efeito inseticida, por meio de possíveis efeitos aditivos ou sinérgicos. Tal combinação, em hipótese, pode reduzir a incidência de populações resistentes para ambas as técnicas de controle, por meio da combinação de diferentes mecanismos de ação; minimizar as limitações do uso de terra de diatomácea, principalmente da poeira nas estruturas de armazenagem por meio da redução das doses utilizadas e reduzir a taxa de degradação de substâncias naturais fotoinstáveis, por meio de um possível efeito protetor dos pós inertes. Assim, objetivou-se avaliar o efeito da associação de extratos orgânicos brutos de anonáceas neotropicais com terra de diatomácea no controle de S. zeamais em milho armazenado. Além da ação inseticida dos tratamentos isolados ou em combinação, também foi avaliado o efeito transgeracional dos mesmos sobre o gorgulho-do-milho.

\section{MATERIALE MÉTODOS}

\section{Insetos}

Os insetos utilizados nos bioensaios foram obtidos de uma população mantida em laboratório sob condições controladas (temperatura de $25 \pm 2^{\circ} \mathrm{C}$, umidade relativa 
de $60 \pm 10 \%$, fotofase de 14 h e luminosidade média de 172 lux), em frascos de vidro com capacidade para três litros, com a boca vedada com tecido fino (voile). Grãos de trigo foram utilizados como substrato para a manutenção da criação de $S$. zeamais, os quais foram expostos, previamente, a $-10^{\circ} \mathrm{C}$, por pelo menos $48 \mathrm{~h}$, em um congelador doméstico, com vistas a eliminar insetos contaminantes (Silva-Aguayo et al., 2006).

\section{Obtenção das espécies e preparação dos extratos orgânicos brutos}

As sementes utilizadas na preparação dos extratos foram obtidas de frutos maduros, coletados em 22/01/2010, em plantas de duas espécies de anonáceas cultivadas no Campus da ESALQ/USP, em Piracicaba, SP (Lat.: 22042'29”S, Long.: 47037'36”'O). Após a coleta, foi obtida uma exsicata de cada uma das espécies, as quais foram encaminhadas aos Drs. Heimo Rainer (Department of Systematics and Evolution of Higher Plants, University of Vienna) e Renato Mello-Silva (Instituto de Biociências, USP) para confirmação específica. Além disso, voucher especimens foram depositados no Museu E.S.A. do Departamento de Ciências Biológicas da ESALQ/USP, em Piracicaba, SP, sob números 121203 (A. montana) e 120985 (A. mucosa).

Para a preparação dos extratos, as sementes foram desidratadas em estufa a $40^{\circ} \mathrm{C}$, por 48 a $72 \mathrm{~h}$. Posteriormente, as amostras foram moídas em um moinho de facas, obtendo-se os pós vegetais, que foram armazenados, separadamente por espécie, em vidros hermeticamente fechados até a sua utilização. Os extratos orgânicos foram obtidos por maceração, utilizandose como solventes [na proporção de $5: 1(\mathrm{p} / \mathrm{v})$, grau análise] em ordem crescente de polaridade, o hexano, o diclorometano e o etanol. Para cada solvente, foi feita a extração até a exaustão, utilizando-se a seguir o solvente de polaridade imediatamente superior. A cada troca de solvente, filtrou-se o macerado através de papel filtro, e eliminou-se o solvente da amostra remanescente em rotaevaporador a $40^{\circ} \mathrm{C}$ e à pressão de -600 mmHg. Após a completa evaporação do solvente em câmara com fluxo de ar, determinou-se o rendimento da extração para cada espécie estudada, nos distintos solventes utilizados.

\section{Bioensaios}

Todos os bioensaios foram conduzidos em sala climatizada à temperatura de $25 \pm 2^{\circ} \mathrm{C}$, umidade relativa de $60 \pm 10 \%$, fotofase de 14 h e luminosidade média de 172 lux. Como substrato para realização dos testes, utilizaram-se grãos de milho inteiros do híbrido AG 1051 (dentado amarelo, semiduro), selecionados manualmente. O delineamento experimental adotado foi o inteiramente aleatorizado.

\section{Avaliação da atividade inseticida dos tratamentos isolados ou em combinação}

Para avaliação da atividade inseticida dos tratamentos isolados ou em combinação, amostras de grãos de milho (50 g), dispostas em potes plásticos de $250 \mathrm{~mL}$ (unidades amostrais), foram submetidas aos seguintes tratamentos: T1 - controle (solvente utilizado na ressuspensão dos extratos); T2 - Extrato hexânico de sementes de $A$. mucosa - EHAmuc (na $\mathrm{CL}_{30}$ estimada); T3 - EHAmuc (na $\mathrm{CL}_{50}$ estimada); T4 - Extrato diclorometânico das sementes de $A$. montana - EDAmon (na $\mathrm{CL}_{30}$ estimada); $\mathrm{T5}$ - EDAmon (na $\mathrm{CL}_{50}$ estimada); T6 - Terra de diatomácea (na $\mathrm{CL}_{30}$ estimada); $\mathrm{T} 7$ - Terra de diatomácea (na $\mathrm{CL}_{50}$ estimada); T8 - Terra de diatomácea (na $\mathrm{CL}_{30}$ ) + EHAmuc (na $\mathrm{CL}_{50}$ estimada); T9 - Terra de diatomácea (na $\mathrm{CL}_{50}$ estimada) + EHAmuc (na $\mathrm{CL}_{30}$ estimada); T10 - Terra de diatomácea (na $\mathrm{CL}_{30}$ estimada) + EDAmon (na $\mathrm{CL}_{50}$ estimada); T11 - Terra de diatomácea (na $\mathrm{CL}_{50}$ estimada) + EDAmon (na $\mathrm{CL}_{30}$ estimada). As CLs foram estimadas em estudos prévios, tanto para os extratos orgânicos brutos (Ribeiro et al., 2013; 2014), quanto para a formulação de terra de diatomácea (Ribeiro et al., 2018).

A formulação comercial utilizada (Keepdry ${ }^{\circledR}$, Irrigação Dias Cruz Ltda., Santo André, SP) é do tipo pó seco (DP) e contém $860 \mathrm{~g} / \mathrm{kg}$ de dióxido de silício $\left(\mathrm{SiO}_{2}\right)$. No Brasil, Keepdry ${ }^{\circledR}$ está registrada para o controle de coleópteros-praga no armazenamento de cevada, feijão, milho e trigo, em doses de 250 a 750 gramas por tonelada (Agrofit, 2019).

Para a aplicação, os extratos foram solubilizados em uma solução de acetona e pulverizados sobre os grãos por meio de microatomizador acoplado a uma bomba pneumática, ajustada para propiciar uma pressão de $0,5 \mathrm{kgf} / \mathrm{cm}^{2}$ e volume de calda de 30 litros por tonelada. Após a pulverização, a mistura dos grãos com os extratos foi feita manualmente, em sacos plásticos com capacidade para dois litros, os quais foram agitados por um minuto. Nas respectivas associações (extratos + terra de diatomácea), os grãos foram submetidos primeiramente ao tratamento com os extratos vegetais e, após a secagem 
dos grãos (duas horas), foi realizado o tratamento com terra de diatomácea, utilizando-se os mesmos procedimentos descritos. Após, cada amostra foi infestada com 50 adultos, não sexados e com idade entre 10 e 20 dias, com seis repetições por tratamento. A mortalidade foi avaliada a cada dois dias até o décimo dia de exposição.

\section{Avaliação do efeito transgeracional (progênie $F_{1}$ )}

As mesmas unidades amostrais do teste anterior foram utilizadas neste bioensaio. Os grãos foram tratados com os respectivos extratos, infestados com 50 adultos não sexados e com idade entre 10 e 20 dias. Decorridos 10 dias da infestação, os adultos foram retirados e as unidades amostrais mantidas nas condições climáticas mencionadas anteriormente. Decorridos 60 dias da infestação inicial, o número de adultos emergidos foi contado em cada unidade amostral. Para cada tratamento foram utilizadas seis repetições.

\section{ANÁLISES DOS DADOS}

Primeiramente, realizou-se um pré-ajuste do modelo normal aos dados e verificou-se a normalidade de resíduos com o teste de Shapiro-Wilk (Shapiro \& Wilk, 1965), bem como a homogeneidade de variâncias com o teste de Bartlett (Bartlett, 1937). Nos casos em que as pressuposições não foram atendidas, prosseguiu-se ao ajuste de um modelo linear generalizado (GLM) pertencente à família exponencial de distribuições (Nelder \& Wedderbum, 1972). A qualidade do ajuste do modelo de GLM foi determinada utilizando-se o gráfico meio normal de probabilidades com envelope de simulação (Demétrio \& Hinde, 1997). Quando houve diferença significativa entre os tratamentos, comparações múltiplas (Tukey post hoc test, $\mathrm{P}<0,05$ ) foram realizadas utilizando a função glht do pacote multicomp, com ajustes dos valores de $p$. Todas as análises foram realizadas utilizando-se o software estatístico R versão 2.13.1 (R Development Core Team, 2012).

\section{RESULTADOS E DISCUSSÃO}

Houve incremento da mortalidade de gorgulhos ( $S$. zeamais) com o aumento do período de exposição aos tratamentos. Todos os tratamentos diferiram do controle após dois dias da aplicação (Tabela 1). De modo geral, o tratamento com terra de diatomácea (248,75 ppm) e a associação de combinações desta com extratos em hexano de sementes de $A$. mucosa $(77,72 \mathrm{ppm})$ foram os tratamentos que ocasionaram as maiores mortalidades, não diferindo entre si em nenhuma das datas de avaliação, mas diferindo da maioria dos demais tratamentos, principalmente nas avaliações feitas a partir dos 6 dias após a aplicação dos produtos (Tabela 1). Por sua vez, a utilização conjunta de terra de diatomácea e extratos de sementes de $A$. mucosa e de $A$. montana resultaram em mortalidades equivalentes ou inferiores em comparação ao emprego desses isoladamente (Tabela 1), o que demonstra efeito antagônico entre esses tratamentos.

Observou-se, ainda, tendência de menor eficácia dos tratamentos empregados em associação com aumento da quantidade de extrato orgânico aplicado. Isso pode ter ocorrido em virtude de que uma fração do extrato vegetal tenha sido adsorvida pelas partículas de terra de diatomácea, com redução da capacidade de ligação dessa fração com os lipídios epicuticulares do inseto, reduzindo sua eficiência, e/ou pela inativação dos compostos bioativos presentes no extrato orgânico. Similarmente, Islam et al. (2010) verificaram que a mistura dos monoterpenoides eugenol e cinamaldeído com terra de diatomácea resultaram em efeitos antagônicos sobre a mortalidade de $C$. maculatus, assim como a utilização da combinação de cinamaldeído e terra de diatomácea no controle de $S$. oryzae. Os mesmos autores verificaram, entretanto, efeitos interativos variáveis, dependendo do monoterpenoide utilizado e da suscetibilidade do inseto-praga alvo. Tais resultados, no entanto, divergem de Athanassiou et al. (2009), que verificaram que a formulação DEBBM-P/WP, composta por terra de diatomácea associada com extratos de raízes de Celastrus angulatus (Celastraceae), foi mais efetiva que a aplicação somente de terra de diatomácea, sugerindo a ocorrência de efeito sinérgico entre as duas técnicas. A natureza e a afinidade química dos compostos presentes nos diferentes extratos pode explicar tais diferenças.

Em relação ao efeito transgeracional dos tratamentos, avaliado por meio do número de insetos emergidos das amostras tratadas (progênie $F_{1}$, Figura 1), foi observada a mesma tendência do efeito letal. Os tratamentos constituídos por terra de diatomácea (ambas as concentrações) e a associação desta com extratos em hexano de sementes de A. mucosa (em ambas as concentrações) ou com o extrato em diclorometano de A. montana $\left(\mathrm{CL}_{50}\right)$ empregado isoladamente, foram os tratamentos que provocaram a maior redução na progênie $F_{1}$ (Figura 1). De modo geral, a associação dos extratos com a formulação de terra de diatomácea 
Tabela 1 - Percentagem de mortalidade acumulada ( \pm erro padrão) de adultos de Sitophilus zeamais expostos a grãos tratados com extratos orgânicos das sementes de Annona mucosa e Annona montana, nas concentrações $\mathrm{CL}_{30} * \mathrm{eCL}_{50}$; com terra de diatomácea, nas concentrações $\mathrm{CL}_{30} \mathrm{eCL}_{50}$ e com a combinação dos extratos com terra de diatomácea.

\begin{tabular}{|c|c|c|c|c|c|c|}
\hline \multirow{3}{*}{$\begin{array}{l}\text { Tratamentos** } \\
\text { Testemunha }\end{array}$} & \multirow{3}{*}{$\begin{array}{c}\begin{array}{c}\text { Concentração) } \\
\text { (ppm }\end{array} \\
-\end{array}$} & \multicolumn{5}{|c|}{ Dias após o tratamento ${ }^{1}$} \\
\hline & & 2 & 4 & 6 & 8 & 10 \\
\hline & & $1,33 \pm 0,66 \quad \mathrm{C}$ & $2,00 \pm 0,89 \quad \mathrm{c}$ & $2,16 \pm 1,04$ & $2,16 \pm 1,04$ e & $2,16 \pm 1,04$ \\
\hline EHAmuc & $77,72\left(=\mathrm{CL}_{30}\right)$ & $7,33 \pm 2,17 \quad b$ & $14,33 \pm 3,24 \mathrm{~b}$ & $17,33 \pm 3,74 \quad d$ & $18,66 \pm 3,71 \quad d$ & $21,66 \pm 3,51$ \\
\hline EHAmuc & $110,28\left(=\mathrm{CL}_{50}\right)$ & $7,00 \pm 2,17 \quad b$ & $17,33 \pm 2,85 b$ & $25,66 \pm 3,15 \mathrm{~cd}$ & $31,33 \pm 3,92 \quad c$ & $38,33 \pm 3,98$ \\
\hline EDAmon & $461,91\left(=\mathrm{CL}_{30}\right)$ & $12,66 \pm 1,97 \mathrm{ab}$ & $24,66 \pm 2,71 \mathrm{ab}$ & $31,66 \pm 3,98$ bc & $34,66 \pm 4,21 \mathrm{c}$ & $35,66 \pm 4,27$ \\
\hline EDAmon & $534,75\left(=\mathrm{CL}_{50}\right)$ & $16,00 \pm 3,05 \mathrm{ab}$ & $34,00 \pm 5,46$ a & $41,33 \pm 4,63 \quad b$ & $47,66 \pm 4,33 \quad b$ & $47,66 \pm 4,33 \mathrm{~b}$ \\
\hline TD & $213,78\left(=\mathrm{CL}_{30}\right)$ & $9,33 \pm 1,22$ & $25,00 \pm 1,77 \mathrm{ab}$ & $36,33 \pm 2,27 \quad$ bc & $39,33 \pm 2,71$ bc & $40,66 \pm 2,56 \mathrm{~b}$ \\
\hline TD & $248,75\left(=\mathrm{CL}_{50}\right)$ & $9,33 \pm 1,33$ & $27,00 \pm 3,13 \mathrm{ab}$ & $51,00 \pm 4,80 \quad a b$ & $58,00 \pm 3,38 \mathrm{ab}$ & $60,16 \pm 3,50 \mathrm{a}$ \\
\hline TD+EHAmuc & $213,78+110,28$ & $13,00 \pm 2,29 \mathrm{ab}$ & $26,66 \pm 3,63 \mathrm{ab}$ & $42,00 \pm 6,69$ & $48,00 \pm 6,92 \quad b$ & $49,83 \pm 6,52$ \\
\hline TD+EHAmuc & $248,75+77,72$ & $10,00 \pm 1,46 \mathrm{ab}$ & $34,66 \pm 2,66$ a & $56,33 \pm 5,35 \quad$ a & $63,33 \pm 5,20 \quad a$ & $67,66 \pm 5,01$ \\
\hline TD+EDAmon & $213,78+534,75$ & $17,33 \pm 3,16$ a & $31,66 \pm 5,04 \mathrm{ab}$ & $34,66 \pm 6,29$ bc & $35,66 \pm 6,60 \quad \mathrm{c}$ & $36,00 \pm 6,85$ \\
\hline TD+EDAmon & $248,75+461,91$ & $11,00 \pm 1,69 \mathrm{ab}$ & $26,00 \pm 2,42 \mathrm{ab}$ & $27,33 \pm 2,76$ & $28,33 \pm 3,07 \mathrm{~cd}$ & $28,33 \pm 3,07 \mathrm{c}$ \\
\hline
\end{tabular}

${ }^{1}$ Médias seguidas de letras distintas, nas colunas, indicam diferença entre tratamentos (GLM com distribuição binomial seguido por teste post hoc de Tukey, $p<0,05)$;

* Aplicadas com um volume de calda de $30 \mathrm{~L} \mathrm{t}^{-1}$;

** EHAmuc: Extrato em hexano de Annona mucosa; EDAmon: Extrato em diclorometano de Annona montana; TD: Terra de diatomácea.

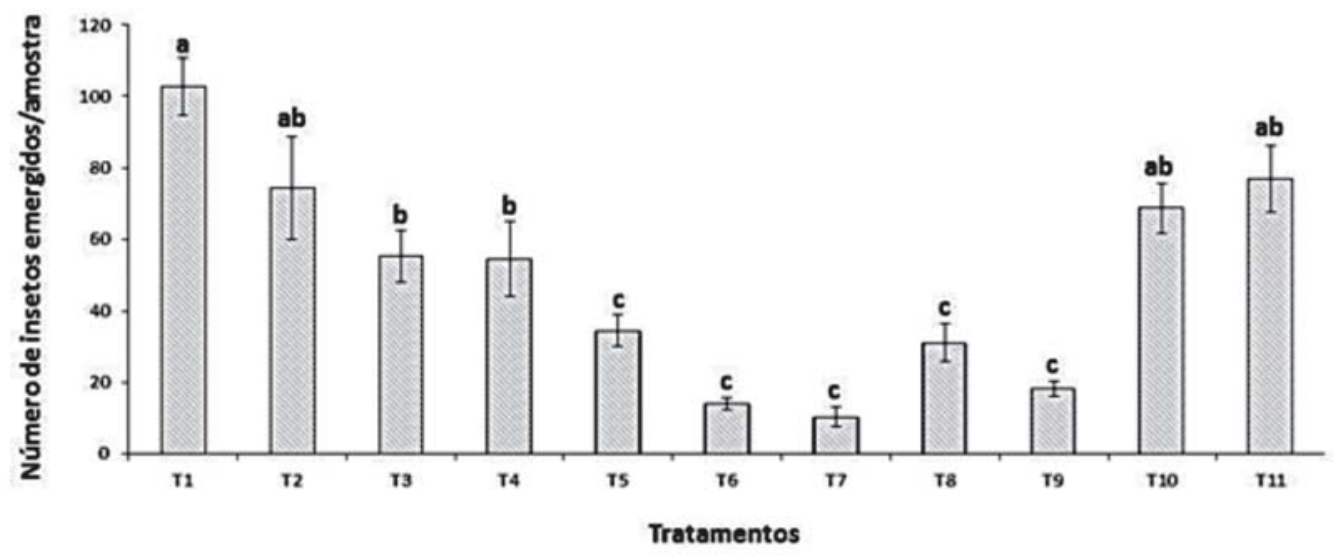

Figura 1 - Médias ( \pm erro padrão) do número de insetos emergidos (progênie F1) após 60 dias da infestação de Sitophilus zeamais, em amostras de grãos tratados com extratos orgânicos das sementes de duas espécies de Annonaceae, nas concentrações $\mathrm{CL}_{30^{*}}$ e $\mathrm{CL}_{50^{*}}$; com terra de diatomácea, nas concentrações $\mathrm{CL}_{30}$ e $\mathrm{CL}_{50}$ e com a combinação entre tais extratos e terra de diatomácea. Nota: T1 - controle (solvente utilizado na ressuspensão dos extratos); T2 - Extrato hexânico de sementes de A. mucosa - EHAmuc (na $\mathrm{CL}_{30}$ ); T3 - EHAmuc (na $\mathrm{CL}_{50}$ ); $\mathrm{T} 4$ - Extrato diclorometânico das sementes de A. montana - EDAmon (na $\mathrm{CL}_{30}$ ); T5 - EDAmon (na $\mathrm{CL}_{50}$ ); T6 - Terra de diatomácea (na $\mathrm{CL}_{30}$ ); T7 - Terra de diatomácea (na $\left.\mathrm{CL}_{50}\right)$; $\mathrm{T} 8$ - Terra de diatomácea $\left(\right.$ na $\left.\mathrm{CL}_{30}\right)+$ EHAmuc $\left(\right.$ na $\mathrm{CL}_{50}$ ); T9 - Terra de diatomácea (na $\left.\mathrm{CL}_{50}\right)+$ EHAmuc (na $\left.\mathrm{CL}_{30}\right)$; T10 - Terra de diatomácea $\left(\mathrm{na} \mathrm{CL}_{30}\right)+$ EDAmon (na $\left.\mathrm{CL}_{50}\right)$; T11 - Terra de diatomácea (na $\mathrm{CL}_{50}$ ) + EDAmon (na $\mathrm{CL}_{30}$ ). Médias seguidas de letras distintas indicam diferenças entre tratamentos (GLM com distribuição de quasi-Poisson seguido por teste post hoc de Tukey, $\mathrm{p}<0,05)$. 
não proporcionou incrementos na redução da progênie $F_{1}$ da referida espécie-praga, o que se configura um efeito antagônico entre as duas técnicas.

Diferentemente dos resultados obtidos, alguns estudos têm demonstrado a possibilidade de associação de pós inertes e óleos essenciais, ocasionando aumento da persistência da atividade inseticida. Assim, Yang et al. (2010) verificaram que a combinação de óleo essencial de Allium sativum (Amaryllidaceae) com terra de diatomácea proporcionou forte efeito aditivo no controle de coleópteros-praga de grãos armazenados. Nguemtchouin et al. (2010), utilizando argila caulinítica como suporte do óleo essencial de Xylopia aethiopica (Dunal) A. Rich (Annonaceae) no preparo de uma formulação inseticida para proteção de grãos de milho contra S. zeamais, observaram que os resultados obtidos com a formulação proposta foram comparáveis ao inseticida Poudrox ${ }^{\circledR}$ (produto sintético à base de malathion). Salienta-se, no entanto, que em virtude da composição predominante de compostos voláteis em óleos essenciais, a adsorção em pós inertes pode ter curta duração e não influenciar a ação inseticida de ambos os produtos. Assim, Nguemtchouin et al. (2010), utilizando monitoramento por headspace, verificaram decréscimos de $91 \%$ para $17 \%$ do teor de óleo essencial de $X$. aethiopica adsorvido em argila caulinítica após 24 h, quando essa foi mantida em caixas abertas. Ulrichs \& Mewis (2000) testaram a combinação de terra de diatomácea e nim, que atua parcialmente como fagodeterrente para $S$. oryzae e $T$. castaneum, e verificaram efeito aditivo entre as duas técnicas quando considerado o número de insetos emergidos (progênie $\mathrm{F}_{1}$ ), fato não observado com os extratos de anonáceas testados nesta pesquisa.

Apesar das possíveis vantagens da associação de estratégias no manejo de insetos-praga de grãos armazenados, tais como a redução da incidência de populações resistentes para ambas as técnicas e o aumento do poder residual de substâncias naturais fotoinstáveis, os resultados obtidos no presente estudo indicam que a utilização conjunta de terra de diatomácea e extratos de sementes de A. mucosa, em hexano, e de $A$. montana, em diclorometano, independentemente da concentração utilizada, ocasionam efeitos inseticidas ou protetores de grãos equivalentes ou mesmo inferiores em comparação aos mesmos produtos, o que demonstra efeito antagônico entre as duas técnicas. Todavia, o aumento das dosagens dos componentes ou mesmo alterações nas proporções de ambos os constituintes podem atenuar tais incompatibilidades e justificar a aplicação conjunta de pós inertes e derivados de anonáceas neotropicais ricos em acetogeninas. Estudos complementares serão necessários para elucidação de tais hipóteses.

\section{LITERATURACITADA}

AGROFIT. Sistema de agrotóxicos fitossanitários. Ministério da Agricultura, Pecuária e Abastecimento - Coordenação Geral de Agrotóxicos e Afins/DFIA/DAS. Disponível em: http://agrofit.agricultura.gov.br/agrofit_cons/ principal_agrofit_cons. Acesso em 01 de fevereiro de 2019.

ANSANTE, T.F.; RIBEIRO, L.P.; BICALHO, K.U. et al. Secondary metabolites from Neotropical Annonaceae: screening, bioguided fractionation, and toxicity to Spodoptera frugiperda (JE Smith) (Lepidoptera: Noctuidae). Industrial Crops and Products, v.74, p.969-976, 2015.

ATHANASSIOU, C.G.; KORUNIC, Z.; VAYIAS, B.J. Diatomaceous earths enhance the insecticidal effect of bitterbarkomycin against stored-grain insects. Crop Protection, v.28, p.123-127, 2009.

ATUI, M.B.; LAZZARI, F.A.; LAZZARI, S.M.N. Avaliação de metodologia para detecção de resíduos de terra de diatomáceas em grãos de trigo e farinha. Revista Instituto Adolfo Lutz, v.62, n.1, p.11-16, 2003.

BARTLETT, M.S. Properties of sufficiency and statistical tests. Proceedings of the Royal Society of London - Series A, v.160, p.268-282, 1937.

BOYER, S.; ZHANG, H.; LEMPÉRIÈRE, G. A review of control methods and resistance mechanisms in stored-product insects. Bulletin of Entomology Research, v.102, p.213-229, 2012.

CERUTTI, F.C.; LAZZARI, S.M.N. Combination of diatomaceous earth and powder deltamethrin for insect control in stored corn. Revista Brasileira de Entomologia, v.49, n.4, p.580-583, 2005. 
DEMÉTRIO, C.G.B., HINDE, J. Half-normal plots and overdispersion. Glim Newsletter, v.27, p.19-26, 1997.

HAGSTRUM, D.W.; PHILLIPS, M.T.W. Evolution of stored-product entomology: protecting the world food supply. Annual Review of Entomology, v.62, p.379-397, 2017.

HIKAL, W.M.; BAESHEN, R.S.; AHL, H.A.H. Botanical insecticide as simple extractives for pest control. Cogent Biology, v.3, 1404274, 2017.

ISLAM, M.F.; HASAN, M.M.; LEI, C. et al. Direct and admixture toxicity of diatomaceous earth and monoterpenoids against the storage pests Callosobruchus maculatus (F.) and Sitophilus oryzae (L.). Journal of Pest Science, v.83, n. 2, p.105-112, 2010.

ISMAN, M.B. Botanical insecticides, deterrents, and repellents in modern agriculture and an increasingly regulated world. Annual Review of Entomology, v.51, p.45-66, 2006.

MALIA, H.A.E.; ROSI-DENADAI, C.A.; CARDOSO, D.G. et al. Dust to weevils, weevils to dust: maize weevil personality and susceptibility to diatomaceous earth. Journal of Pest Science, v.89, n.2, p.469-478, 2016.

MARSARO JÚNIOR, A.L.; MOURÃO JÚNIOR, M.; PAIVA, W.R.S.C. et al. Eficiência da terra de diatomácea no controle de Sitophilus zeamais em milho armazenado. Revista Acadêmica de Ciências Agrárias e Ambientais, v.5, n.1, p.27-32, 2007.

NELDER, J.A.; WEDDERBURN, R.W.M. Generalized linear models. Journal of the Royal Statistical Society, v.135, p.370-384, 1972.

NGUEMTCHOUIN, M.M.G.; NGASSOUM, M.B.; NGAMO, L.S.T. et al. Insecticidal formulation based on Xylopia aethiopica essential oil and kaolinite clay for maize protection. Crop Protection, v.29, p.985-991, 2010.

OLIVEIRA, J.V.; VENDRAMIM, J.D. Repelência de óleos essenciais e pós vegetais sobre adultos de Zabrotes subfasciatus (Boh.) (Coleoptera:
Bruchidae) em sementes de feijoeiro. Anais da Sociedade Entomológica do Brasil, v.28, n.3, p.549-555, 1999.

PINHEIRO, M.L.B.; XAVIER, C.M.; SOUZA, A.D.L. et al. Acanthoic acid and other constituents from the stem of Annona amazonica (Annonaceae). Journal of the Brazilian Chemical Society, v.20, n.6, p.1095-1102, 2009.

R DEVELOPMENT CORE TEAM. R: A language and environment for statistical computing. $\mathrm{R}$ Foundation for Statistical Computing, Vienna, Austria, 2012.

RIBEIRO, L.P.; COSTA, E.C.; KARLEC, F.; BIDINOTO, V.M. Avaliação da eficácia de pós inertes minerais no controle de Sitophilus zeamais Mots. (Coleoptera: Curculionidae). Revista da FZVA, v.15, n.2, p.19-27, 2008.

RIBEIRO, L.P.; LOVATTO, M.; VENDRAMIM, J.D. Avaliação da eficácia de duas formulações comerciais de terra de diatomácea no controle do gorgulho-do-milho com base em parâmetros toxicológicos. Agropecuária Catarinense, v.31, n.1, p.56-60, 2018.

RIBEIRO, L.P.; VENDRAMIM, J.D.; ANDRADE, M.S. et al. Tropical plant extracts as sources of grainprotectant compounds against Sitophilus zeamais Motschulsky (Coleoptera: Curculionidae).

Neotropical Entomology, v.43, p.470-482, 2014.

RIBEIRO, L.P.; VENDRAMIM, J.D.; BICALHO, K.U. et al. Annona mucosa Jacq. (Annonaceae): a promising source of bioactive compounds against Sitophilus zeamais Mots. (Coleoptera:

Curculionidae). Journal of Stored

Products Research, v.55, p.6-14, 2013.

RIBEIRO, L.P.; VENDRAMIM, J.D.; GONÇALVES, G.L.P. et al. Searching for promising sources of grain protectors in extracts from Neotropical Annonaceae. Boletín Latinoamericano y del Caribe de Plantas Medicinales y Aromaticas, v.15, p.215-232, 2016.

ROSELL, G.; QUERO, C.; COLL-TOLEDANO, J.; GUERRERO, A. Biorational insecticides in pest management. Journal of Pesticide Science, v.33, n.2, p.103-121, 2008. 
RUMBOS, C.; DUTTON, A.C.; TSIROPOULOS, N.G.; ATHANASSIOU, C.G. Persistence and residual toxicity of two pirimiphos-methyl formulations on wheat against three storedproduct pests. Journal of Stored Products Research, v.76, p.14-21, 2018.

SHAH, M.A.; KHAN, A.A. Use of diatomaceous earth for the management of stored-product pests.

International Journal of Pest

Management, v.60, n.2, p.100-113, 2014.

SHAPIRO, S.S.; WILK, M.B. An analysis of variance test for normality (complete samples).

Biometrika, v.52, v.3/4, p.591-611, 1965.

SILVA-AGUAYO, G.; GALLO, R.H.; VARGAS, M.T. et al. Evaluación de boldo (Peumus boldus Molina) y cal para el control de Sitophilus zeamais Motschulsky. Agrociencia, v.40, p.219228, 2006.
SOUZA, C.M.; BALDIN, E.L.L.; RIBEIRO, L.P. et al. Lethal and growth inhibitory activities of Neotropical Annonaceae-derived extracts, commercial formulation, and an isolated acetogenin against Helicoverpa armigera.

Journal of Pest Science, v.90, p.701-709, 2017.

ULRICHS, C.; MEWIS, I. Controlling the stored product pests Sitophilus oryzae and Tribolium castaneum by contaminating rice with neem and diatomaceous earth. Anzeiger für Schädlingskunde, v.73, p.37-40, 2000.

YANG, F.L.; LIANG, G.W.; XU, Y.J. et al. Diatomaceous earth enhances the toxicity of garlic, Allium sativum, essential oil against stored-product pests. Journal of Stored Products Research, v.46, p.118-123, 2010.

Recebido para publicação em 8/1/2019 e aprovado em 25/3/2019. 